$\xi=-$ 国

\title{
Design and software implementation of solid state transformer
}

\author{
Dr. Raaed Faleh Hassan * \\ Department of Control and Automation Engineering Techniques \\ Electrical Engineering Technical College \\ Middle Technical University \\ *Corresponding author E-mail: drraaed_alanbaki@eetc.mtu.edu.iq
}

\begin{abstract}
The work presented in this paper concerned with the analysis, design and software implementation of the Solid State Transformer as an alternative to the conventional power transformer. The proposed transformer aims to perform the same task as the conventional one with additional facilities and advantages. Three stages are considered to configure the Solid State Transformer. The first stage which is known as input stage and implemented using Vienna rectifier which converts the AC voltage of the main supply to a DC voltage. The second stage (isolation stage) step down the DC voltage to a lower level DC voltage. This stage consists of a single - phase five-level diode clamped inverter, $1 \mathrm{KHz}$ step - down transformer and fully controlled bridge rectifier. The output stage (third stage) is a three-phase three-level diode clamped inverter which converts the low level DC voltage to a three-phase, $50 \mathrm{~Hz}$ AC voltage. Model Predictive Current Control has been employed for driving transformer's stages. The gating signal is produced directly when the given cost function is minimized, therefore there is no need of any modulator. Behavior of the proposed structure is achieved by simulation which shows high quality power conversion with low Total Harmonic Distortion.
\end{abstract}

Keywords: Solid-State Transformer; Model Predictive Current Control; Vienna Rectifier; Diode Clamped Converter and High Frequency Transformer.

\section{Introduction}

Power transformer plays important role in the electric power systems. It is enables these systems to transfer the generated electrical power for long distance with high efficiency [1-4]. In order to achieve low transmission losses, power transformers providing voltage boosting at the generation side of the power system. However, at the distribution side, another power transformer acts to stepping down the voltages to the values used by industrial, commercial, and residential applications [4]. Although it is important part in the power systems, power transformer has some drawbacks like bulky size, heavy weight, sensitive to the load variations, unable to compensate power factor, etc. [1-3], [5-7]. Power transformers dominate $25 \%$ of the overall size of the power system and more than $30 \%$ of the overall weight. The size of the power transformer is determined by the value of the saturation flux density of the material implements the transformer core. As the maximum operational flux density is inversely proportional with the operating frequency, then increasing this frequency will lead to reducing the size of the transformer. With continuous advancement of power electronics devices and circuits, a considerable possibility to develop promising technique that performs the function of the power transformer and overcomes the transformer drawbacks became at hand The technique that has attracted researcher's attention as an alternative to the conventional transformer is the Solid State Transformer (SST) or Power Electronics Transformer (PET) [2]. In addition to performing the same task as traditional transformer, employing SST opens up a new horizon in the utilization of electrical power systems [5]. The proposed structure of the SST allows of adopting high operating frequency which leads to valuable reduction in the weight and size of this new transformer [8-11]. SST enables a perfect isolation of induced harmonics from passing through power system [1]. It is also performing power conversion between different formats with any desired frequencies [12], keeps instantaneous voltage regulation [13], reactive power compensation, and potential current limitation [10]. SST can be considered as the backbone of the future intelligent power systems [13]. The major task of the SST is to perform the voltage stepping up/down based on medium - to - high frequency isolation, therefore to achieve a considerable reduction in its size and weight. In order to realize this task, the $50 / 60 \mathrm{~Hz}$ ac voltage is transformed to a high frequency by means of rectifier / inverter set. Then this high frequency ac voltage is either stepped up or down using high frequency transformer. Finally, the output of the high frequency transformer is shaped back into $50 / 60 \mathrm{~Hz}$ through another rectifier / inverter set [4]. The presence of the rectifiers and inverters in the composition of these transformers provides the possibility to achieve voltage and current regulation, power flow control, fault current limitation. Also the configuration of the SST provides a scope to supply different voltage forms ( $\mathrm{ac}$ and dc) at the load side which enables the new era in the power systems. Penetration of the SST into distribution power systems needs its structure to employ power devices which have the ability to work reliably in high voltages and frequencies environment [4]. Recently, the researcher's efforts concerned with adopting either multi - modules for the SST structure [1] [5-7] [11] [1519], or using multilevel configuration [2] [12]. Employing the high voltage semiconductor devices based on wide - bandgap substances like $4 \mathrm{H}-\mathrm{SiC}$, leads to the appearance of the third version of the SST configuration [9] [13] [20-21]. Various control methods are presented for controlling the power converters, and Fig. (1) summarize the most familiar ones [22]. 


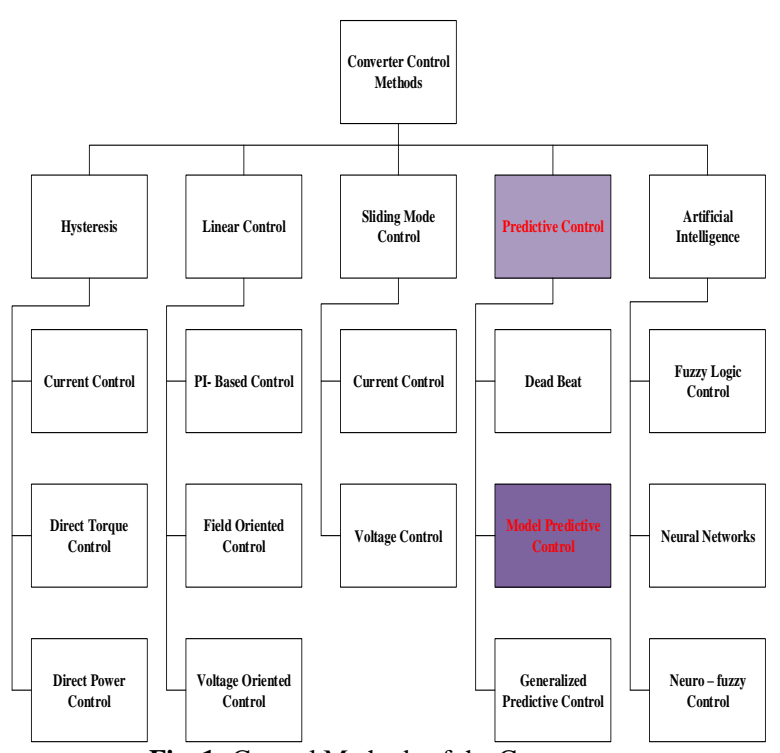

Fig. 1: Control Methods of the Converters.

From these control pattern, predictive scheme seems to be a most interesting method for controlling power converters [22-27]. The main feature of the Model Predictive Control (MPC) is the utilization of the system model to predict the future response of the controlled variable [22]. The conventional control techniques adopted in power electronics drives are based on providing a suitable control signal to the modulator using Proportional - Integral (PI) controller [27]. With the presence of the control signal from the PI controller, the modulator tends to direct the switching states of the converter. Alternatively, MPC combines the two driving stages into a single computational stage that manipulates the discrete and finite switching states of the converter [28].

\section{Basic SST structure}

Between several structures have been proposed for the SSTs, the three - stages structure is the most assessed one [29-32]. Fig.2 shows the three stages SST topology configuration proposed for distribution system, the three stages are namely the input stage, isolation stage and output stage [31].

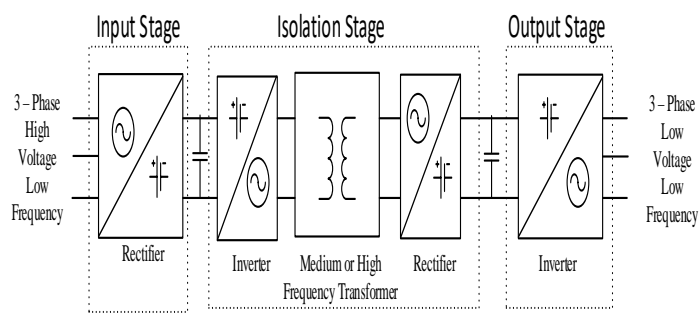

Fig. 2: A Three - Stages SST Topology for Distribution System.

From Fig.2, the input stage in the SST structure acts as a rectifier, which converts the three phase ac voltage to the dc voltage. In the distribution system, the rectifier will be interface with the high or medium ac voltage levels [32]. Therefore, there is a necessity to employ a structure that has a capability of handling these voltage levels and keeps acceptable power quality. Vienna rectifier is one of the most widely considered structure in the recent years [33]. The conventional Vienna structure consists of one active switch per phase; it possesses an advantages over the classical rectifiers structures from power quality and high voltage level points of view. In order to reduce the voltage stress on the components of Vienna rectifier, a proposed structure which is shown in Fig. 3 is considered as the input stage of the SST.

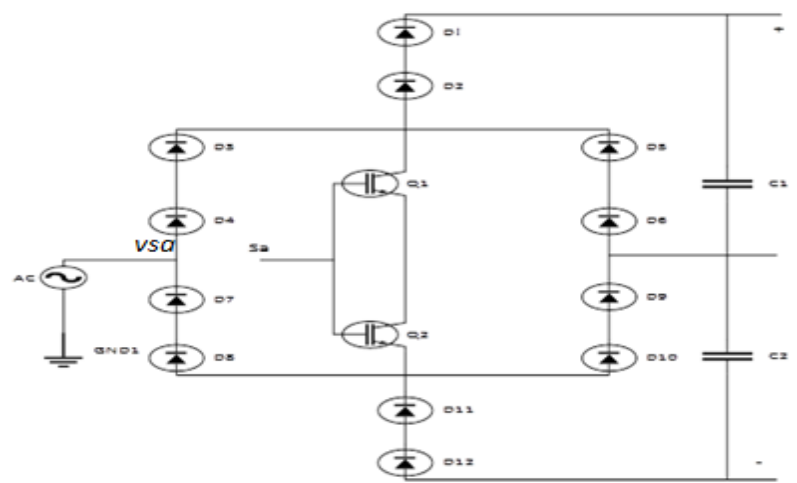

Fig. 3: Proposed One Leg Vienna Structure (Input Stage of the SST).

In this topology, the phase voltage $v_{s a}$ at the (ac) side of the rectifier depends on the polarity of the line current $i_{s a}$ and the switches state $s_{a}$ [33]. When the switches are on the ON state $\left(s_{a}=1\right)$, the center point of the DC side will be connected to the phase voltage $v_{s a}$. However, when the switches are on the OFF state $\left(s_{a}=0\right)$, the phase voltage $v_{s a}$ will be connected to the positive or negative DC bus according to the polarity of the line current $i_{s a}$ [33].

The second stage in the SST structure is the isolation stage which is shown in Fig.4 and configured as a DC - DC converter. In this stage, the high voltage DC input is converted to the single phase AC medium voltage high frequency using Diode Clamped Multilevel Converter (DCMC). A single phase high frequency transformer will transform the medium voltage into low voltage level, and the output of the transformer then rectified to a low level DC voltage using fully controlled bridge rectifier. For the five level diode clamped inverter, the steps to configure the five level voltages are shown in table 1 .

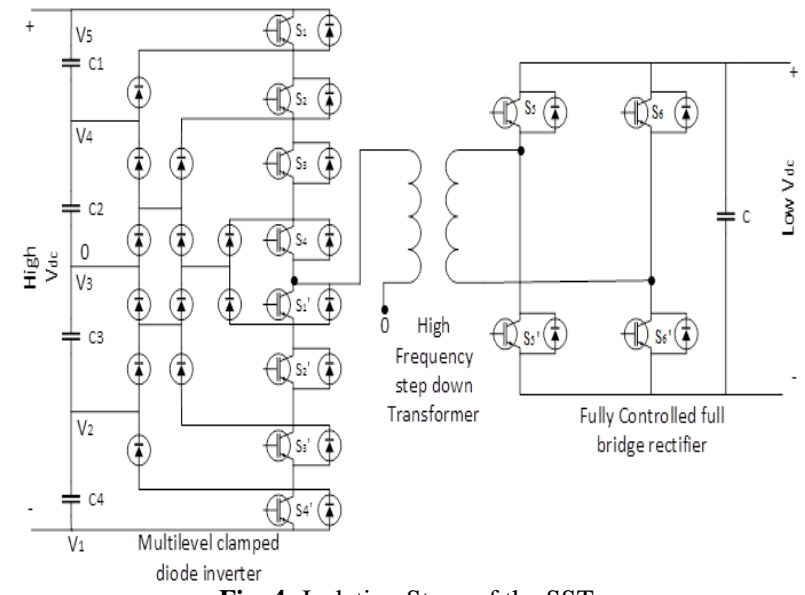

Fig. 4: Isolation Stage of the SST.

Table 1: The Switching States of Five Level Diode Clamped Inverter

\begin{tabular}{lllllllll}
\hline $\mathrm{V}_{\mathrm{o}}$ & $\mathrm{S}_{1}$ & $\mathrm{~S}_{2}$ & $\mathrm{~S}_{3}$ & $\mathrm{~S}_{4}$ & $\mathrm{~S}_{1}^{\prime}$ & $\mathrm{S}_{2}^{\prime}$ & $\mathrm{S}_{3}^{\prime}$ & $\mathrm{S}_{4}^{\prime}$ \\
\hline $\mathrm{V}_{\mathrm{dc}}$ & 1 & 1 & 1 & 1 & 0 & 0 & 0 & 0 \\
$\mathrm{~V}_{\mathrm{dc}} / 2$ & 0 & 1 & 1 & 1 & 1 & 0 & 0 & 0 \\
0 & 0 & 0 & 1 & 1 & 1 & 1 & 0 & 0 \\
$-\mathrm{V}_{\mathrm{dc}} / 2$ & 0 & 0 & 0 & 1 & 1 & 1 & 1 & 0 \\
$-\mathrm{V}_{\mathrm{dc}}$ & 0 & 0 & 0 & 0 & 1 & 1 & 1 & 1 \\
\hline
\end{tabular}

The third stage in the SST structure is the output stage which is responsible of converting the low level DC voltage from the high frequency transformer into low level low frequency 3 - phase AC voltage using another DCMC as shown in Fig.5. 


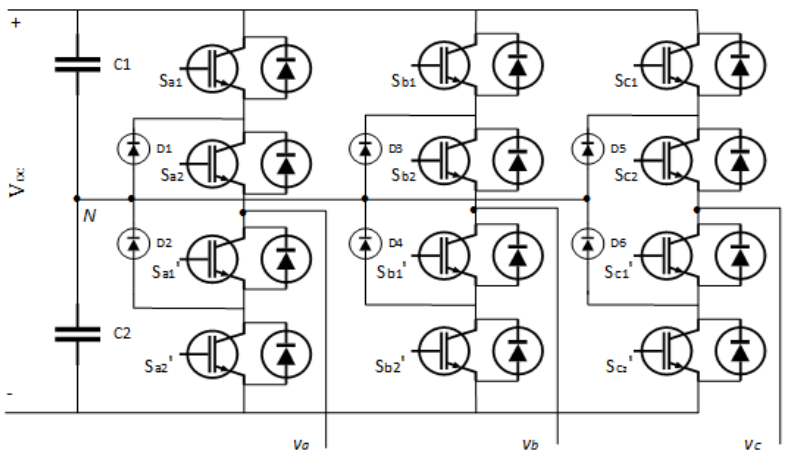

Fig. 5: Output Stage of the SST.

For the three level diode clamped inverter, the steps to configure the three level voltages are shown in table 2.

Table 2: The Switching States of Three-Level Diode Clamped Inverter

\begin{tabular}{lllllllll}
$\mathrm{V}_{\mathrm{an}}$ & $\mathrm{V}_{\mathrm{bn}}$ & $\mathrm{V}_{\mathrm{cn}}$ & $\mathrm{S}_{\mathrm{a} 1}$ & $\mathrm{~S}_{\mathrm{a} 2}$ & $\mathrm{~S}_{\mathrm{b} 1}$ & $\mathrm{~S}_{\mathrm{b} 2}$ & $\mathrm{~S}_{\mathrm{c} 1}$ & $\mathrm{~S}_{\mathrm{c} 2}$ \\
\hline $\mathrm{V}_{\mathrm{dc}}$ & 0 & 0 & 1 & 1 & 0 & 0 & 0 & 0 \\
$\mathrm{~V}_{\mathrm{dc}}$ & $\mathrm{V}_{\mathrm{dc}} / 2$ & 0 & 1 & 1 & 0 & 1 & 0 & 0 \\
$\mathrm{~V}_{\mathrm{dc}}$ & $\mathrm{V}_{\mathrm{dc}}$ & 0 & 1 & 1 & 1 & 1 & 0 & 0 \\
$\mathrm{~V}_{\mathrm{dc}} / 2$ & $\mathrm{~V}_{\mathrm{dc}}$ & 0 & 0 & 1 & 1 & 1 & 0 & 0 \\
0 & $\mathrm{~V}_{\mathrm{dc}}$ & 0 & 0 & 0 & 1 & 1 & 0 & 0 \\
0 & $\mathrm{~V}_{\mathrm{dc}}$ & $\mathrm{V}_{\mathrm{dc}} / 2$ & 0 & 0 & 1 & 1 & 0 & 1 \\
0 & $\mathrm{~V}_{\mathrm{dc}}$ & $\mathrm{V}_{\mathrm{dc}}$ & 0 & 0 & 1 & 1 & 1 & 1 \\
0 & $\mathrm{~V}_{\mathrm{dc}} / 2$ & $\mathrm{~V}_{\mathrm{dc}}$ & 0 & 0 & 0 & 1 & 1 & 1 \\
0 & 0 & $\mathrm{~V}_{\mathrm{dc}}$ & 0 & 0 & 0 & 0 & 1 & 1 \\
$\mathrm{~V}_{\mathrm{dc}} / 2$ & 0 & $\mathrm{~V}_{\mathrm{dc}}$ & 0 & 1 & 0 & 0 & 1 & 1 \\
$\mathrm{~V}_{\mathrm{dc}}$ & 0 & $\mathrm{~V}_{\mathrm{dc}}$ & 1 & 1 & 0 & 0 & 1 & 1 \\
$\mathrm{~V}_{\mathrm{dc}}$ & 0 & $\mathrm{~V}_{\mathrm{dc}} / 2$ & 1 & 1 & 0 & 0 & 0 & 1 \\
& & & & & & & &
\end{tabular}

\section{Control strategy}

The control strategy considered in this paper will be the Model Predictive Current Control (MPCC), which has the general scheme shown in Fig.6. The main feature of the MPCC is the utilize of the system model for predicting the subsequent response of the controlled variables $\mathrm{X}(\mathrm{k}+1)$. This information is employed to optimize the next actuation, according to the minimization of a predefined cost function [23]. In the case of converter, the prediction is performed for each possible switching states. The switching state that minimizes the cost function is applied to the converter in the next sampling interval. The cost function to be minimized is the error between the reference currents and the predicted values in the orthogonal coordinates form as given below:

$g_{\text {cost }}=\left|i_{\alpha}^{*}(k+1)-i_{\alpha}^{p}(k+1)\right|+\left|i_{\beta}^{*}(k+1)-i_{\beta}^{p}(k+1)\right|$

Where $i_{x}^{*}$ is the reference current and $i_{x}^{p}$ is the predicted value.

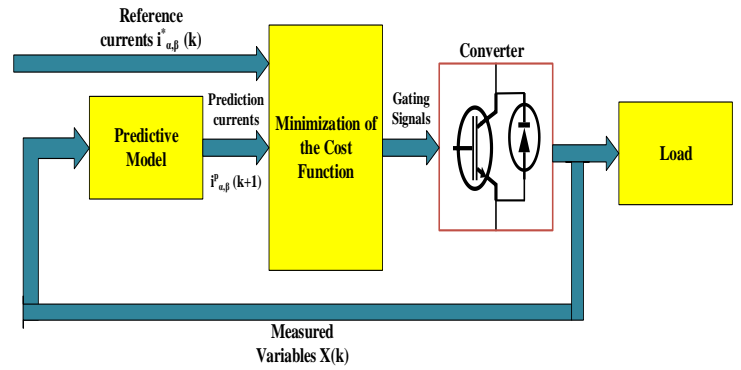

Fig. 6: Generalized MPCC Scheme.

The predictive model block determines the future values of the currents in the orthogonal form $\left(\mathrm{I}^{\mathrm{P}}{ }_{\mathrm{A}} \mathrm{I}_{\mathrm{B}}^{\mathrm{P}}\right)$ for each switching state of the converter. These predictions will be compared with the reference currents to determine the best switching state to be applied to the converter in the next sampling interval. In comparison with the conventional control strategies, MPCC is more simple in its structure but needs more calculation steps.

a) MPCC for Input Stage:
Consider the circuit shown in Fig.7, KVL for each phase can be written as:

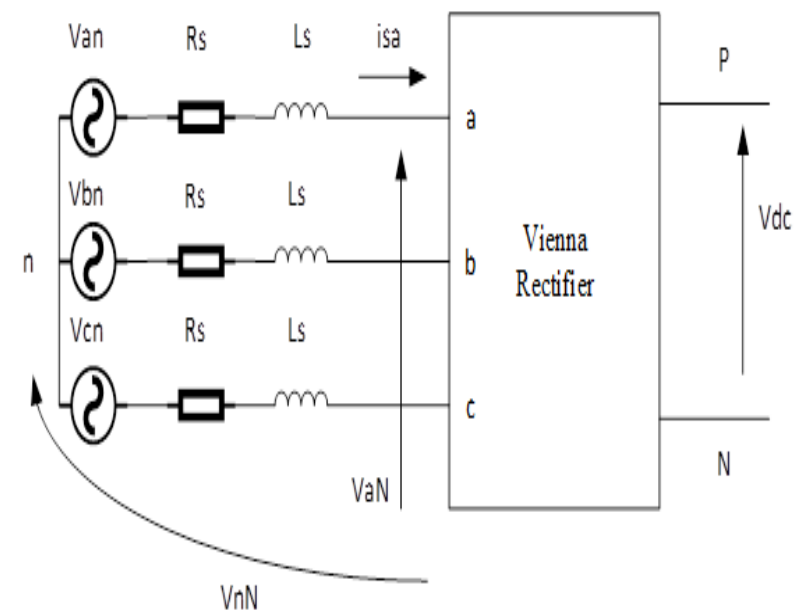

Fig. 7: Vienna Rectifier.

$v_{s a}=L_{s} \frac{d i_{s a}}{d t}+R_{s} i_{s a}+v_{a N}-v_{n N}$

$v_{s b}=L_{s} \frac{d i_{s b}}{d t}+R_{s} i_{s b}+v_{b N}-v_{n N}$

$v_{s c}=L_{s} \frac{d i_{s c}}{d t}+R_{s} i_{s c}+v_{c N}-v_{n N}$

The space vector of the grid voltage can be described as follows:

$V_{s}=\frac{2}{3}\left(v_{s a}+a v_{s b}+a^{2} v_{s c}\right)$

Substitute $(2-4)$ in 5 yield:

$V_{s}=L_{s} \frac{d i_{s}}{d t}+R_{s} i_{s}+V_{a f e}$

Where:

$a=e^{j 2 \pi / 3}$.

And

$\boldsymbol{i}_{\boldsymbol{s}}=\frac{2}{3}\left(i_{s a}+a i_{s b}+a^{2} i_{s c}\right)$

$\frac{2}{3}\left(v_{n N}+a v_{n N}+a^{2} v_{n N}\right)=0$

$V_{a f e}=\frac{2}{3}\left(v_{a N}+a v_{b N}+a^{2} v_{c N}\right)$

$=\frac{2}{3} V_{d c}\left(s_{a}+a s_{b}+a^{2} s_{c}\right)$

Where $s_{a}, s_{b}$, and $s_{c}$ are the switching states of the rectifier.

Equation (6) can be discretized using forward Euler method which yields:

$i_{s}(k+1)=\left(1-\frac{R_{s} T_{S}}{L_{s}}\right) i_{s}(k)+\frac{T_{S}}{L_{s}}\left[V_{S}(k)-V_{a f e}\right]$

Where $i_{s}(k+1)=i_{s \alpha}(k+1)+j i_{s \beta}(k+1)$ is the prediction of the line current vector and $T_{s}$ is the sampling interval. Fig. (8) shows the predictive current control of the input stage. The cost function to be minimized is defined as follows:

$g_{\text {rect. }}=\left|i_{s \alpha}^{*}(k)-i_{s \alpha}(k+1)\right|+\left|i_{s \beta}^{*}(k)-i_{s \beta}(k+1)\right|$ 


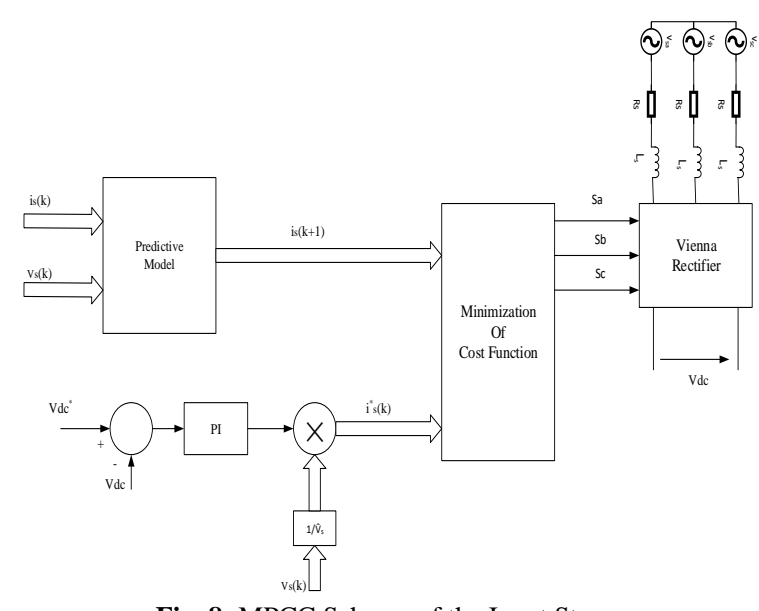

Fig. 8: MPCC Scheme of the Input Stage.

b) MPCC for Isolation Stage

This section presents a MPCC of the isolation stage which is shown in Fig.9. At the inverter side, the prediction is performed for currents and the cost function is defined as:

$$
g_{\text {invhf }}=\left|i_{\alpha}^{*}(k+1)-i_{\alpha}^{p}(k+1)\right|+\left|i_{\beta}^{*}(k+1)-i_{\beta}^{p}(k+1)\right|
$$

While at the rectifier side the active and reactive power are directly controlled when the following cost function is minimized:

$$
g_{\text {rectifier }}=\left|P_{i n}^{*}-P_{i n}(k+1)\right|+\left|Q_{i n}^{*}-Q_{i n}(k+1)\right|
$$

Where, $P_{i n}^{*}$ and $Q_{i n}^{*}$ are the desired active and reactive power respectively, $P_{i n}(k+1)$ and $Q_{i n}(k+1)$ are the predicted active and reactive power respectively. As a unity power factor is required the desired reactive power $Q_{i n}^{*}$ is set equal to zero. The predicted active and reactive power can be calculated as follows

$$
\begin{aligned}
& P_{\text {in }}(k+1)=\operatorname{Re}\left\{v_{s}(k+1) \bar{\iota}_{s}(k+1)\right\}=v_{s \alpha} i_{s \alpha}+v_{s \beta} i_{s \beta} \\
& Q_{\text {in }}(k+1)=\operatorname{Im}\left\{v_{s}(k+1) \bar{l}_{s}(k+1)\right\}=v_{s \beta} i_{s \alpha}-v_{s \alpha} i_{s \beta}
\end{aligned}
$$

Where, $\overline{i_{s}}(k+1)$ is the complex conjugate of $i_{s}(k+1)$.

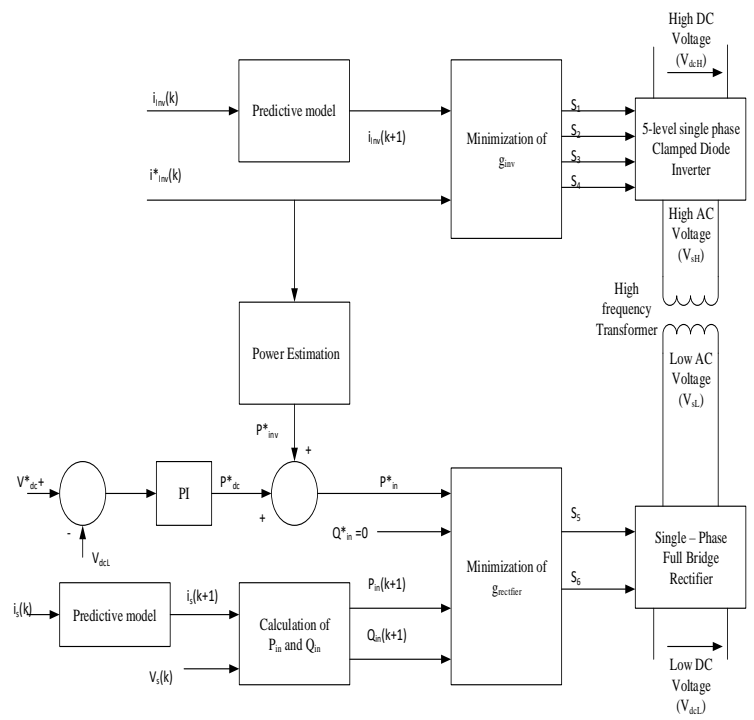

Fig. 9: MPCC Scheme for the Isolation Stage.

c) MPCC for the output stage:

The MPCC scheme of the output stage is shown in Fig. (10).

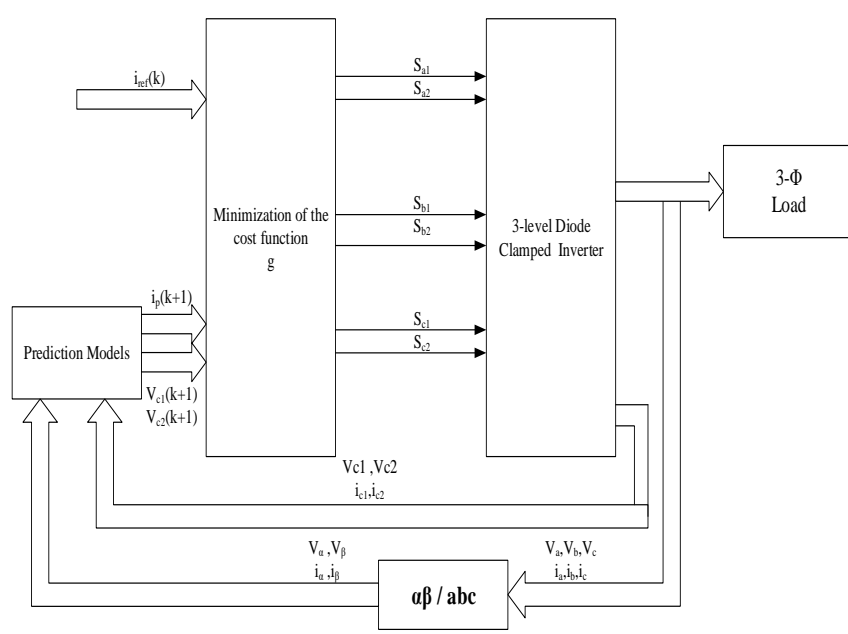

Fig. 10: MPCC for the Output Stage.

The prediction process of the load currents and capacitor voltages is accomplished for the switching state depicted in table II by means of the following equations:

$\hat{e}(k-1)=v(k-1)-\frac{L}{T_{s}} i(k)-\left(R-\frac{L}{T_{s}}\right) i(k-1)$

$i^{P}(k+1)=\left(1-\frac{R T_{s}}{L}\right) i(k)+\frac{T_{s}}{L}(v(k)-\hat{e}(k))$

$v_{c 1}^{P}(k+1)=v_{c 1}(k)+\frac{1}{c} i_{c 1}(k) T_{s}$

$v_{c 2}^{P}(k+1)=v_{c 2}(k)+\frac{1}{c} i_{c 2}(k) T_{s}$

After acquiring the predictions, the cost function is assessed as follows

$g_{\text {invlf }}=\left|i_{\alpha}^{*}-i_{\alpha}^{P}\right|+\left|i_{\beta}^{*}-i_{\beta}^{P}\right|+\left|v_{c 1}^{P}-v_{c 2}^{P}\right|$

The switching state that minimizes the $g_{\text {invlf }}$ is selected to be applied in the next period.

\section{Simulation results}

To confirm the expected behavior of the proposed SST, the designed structure has been simulated using Matlab - Simulink software package. The simulated configuration of the SST is that shown in Fig. 11, which is consist of the three stages have been designed previously.

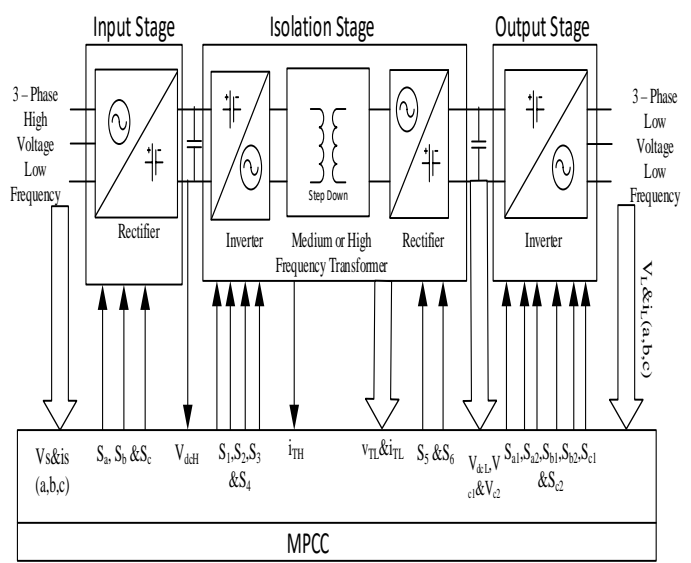

Fig. 11: Simulated Configuration of the SST.

Table 3 shows the designed parameters for each stage in the SST. 
Table 3: Design Parameters of SST

\begin{tabular}{lll}
\hline Input Stage & Isolation Stage & Output Stage \\
\hline Supply voltage: & Input voltage: & Input voltage $: 650 \mathrm{~V}$ \\
$11 \mathrm{KV}(\mathrm{L}-\mathrm{L}$ rms, $50 \mathrm{~Hz})$ & $2.6 \mathrm{KV} \mathrm{DC}$ & $\mathrm{DC}$ \\
Output voltage: $2.6 \mathrm{KV}$ & Output voltage : & Output voltage $: 400 \mathrm{~V}$ \\
$\mathrm{DC}$ & $650 \mathrm{~V} \mathrm{DC}$ & $(\mathrm{L}-\mathrm{L}, \mathrm{rms}, 50 \mathrm{~Hz})$ \\
$\mathrm{R}_{\mathrm{s}}: 5 \Omega, \mathrm{L}_{\mathrm{s}}: 2 \mathrm{mH}, \mathrm{C}_{1}$, & $\mathrm{C}_{1}, \mathrm{C}_{2}, \mathrm{C}_{3}, \mathrm{C}_{4}, \mathrm{C}$ & $\mathrm{C}_{1}, \mathrm{C}_{2}: 5000 \mu \mathrm{F}$ \\
$\mathrm{C}_{2}: 5000 \mu \mathrm{F}$. & $: 5000 \mu \mathrm{F}$. & \\
$\mathrm{K}_{\mathrm{p}}: 10, \mathrm{~K}_{\mathrm{i}}: 0.01$ & Trans. turn ratio: & Load rating: $10 \mathrm{KW}$, \\
& $2: 1$ & 15 KVAR inductive
\end{tabular}

For the purpose of prediction, the MPCC receives the following measured quantities:

- Instantaneous values of the three - phase supply voltages and currents $\left(\mathrm{v}_{\mathrm{s}} \& \mathrm{i}_{\mathrm{s}}\right)_{\mathrm{a}, \mathrm{b}, \mathrm{c}}$ form the main supply.

- High level DC voltage from the output of the input stage.

- Instantaneous value of the current from the primary side of the High - Frequency Transformer (HFT).

- Instantaneous values of the current and voltage from the secondary side of the HFT.

- Low level DC voltage from the output of the isolation stage and $\mathrm{V}_{\mathrm{c} 1}$ and $\mathrm{V}_{\mathrm{c} 2}$ from the input of the output stage.

- Instantaneous values of the three - phase load voltages and currents $\left(v_{l} \& i_{1}\right)_{a, b, c}$ from the output of the output stage.

According to the aforementioned calculations equations (2-18), the MPCC generates 3 - gating signals to the input stage, 6 - gating signals to the isolation stage and 6 - gating signals to the output stage.

Simulation results of the input stage of the SST is shown in Fig. (12). The input voltage from the main supply is three phase $11 \mathrm{KV}$ line - to - line. The input stage converted this AC voltage to DC voltage of magnitude $2600 \mathrm{~V}$. A unity power factor between the phase voltage $V_{\text {an }}$ and the line current $i_{\text {sa }}$ can be shown in the same figure, also a minimum Total Harmonic Distortion (THD) of the line current is achieved as shown in Fig. (13).
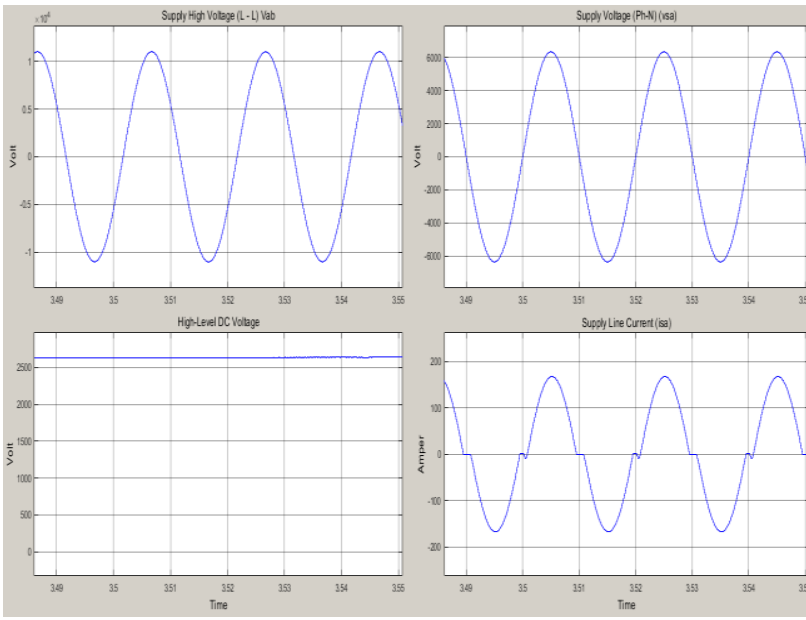

Fig. 12: Simulation Results of the Input Stage.
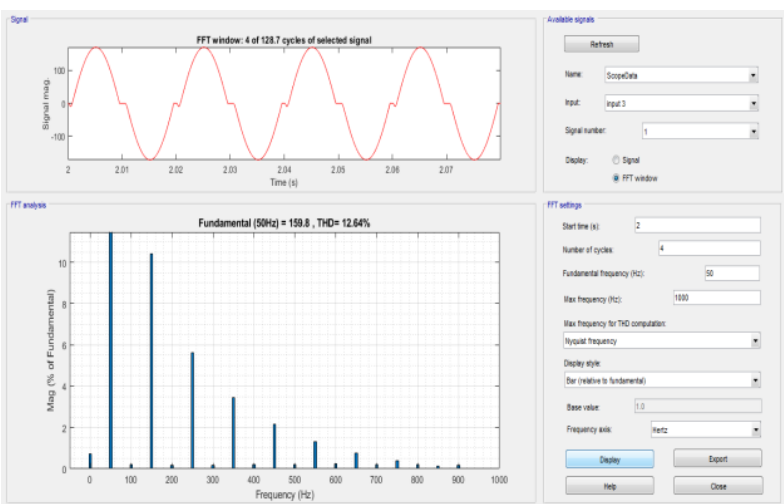

Fig. 13: Total Harmonic Distortion of the Supply Current.

The behavior of the isolation stage is shown in Fig. (14), high level DC voltage produced from the input stage is converted to a high - level high - frequency $(1 \mathrm{KHz}) \mathrm{AC}$ voltage using five level diode clamped converter. High frequency step down transformer is employed to transform the high - level high - frequency AC voltage to the low - level high - frequency AC voltage. Subsequently, a fully controlled bridge rectifier converts the AC voltage to low level DC voltage.

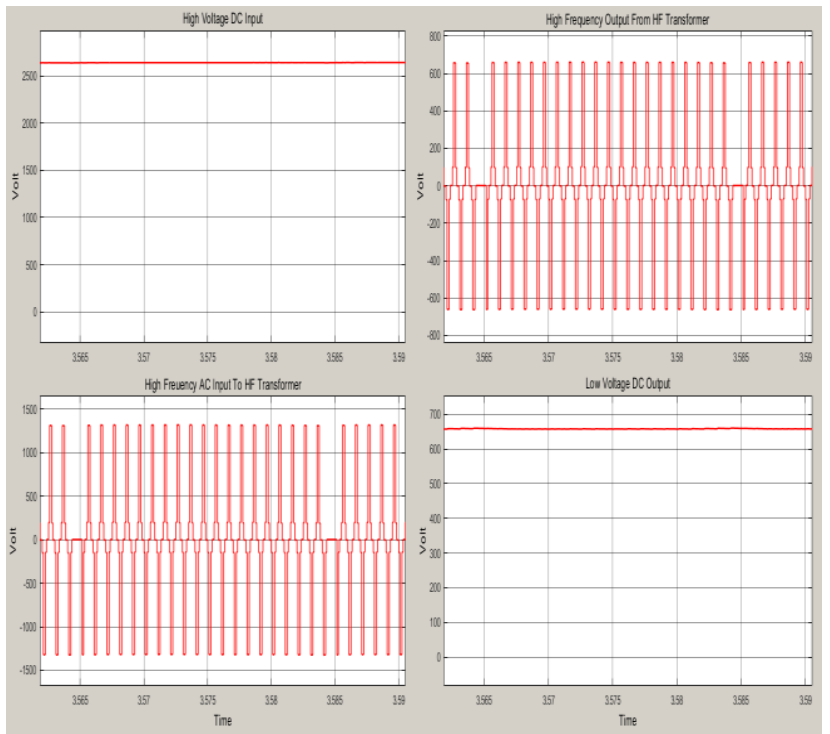

Fig. 14: Behavior of the Isolation Stage.

Simulation results of the output stage are shown in Fig. (15), the low level DC voltage is converted into low level 3-phase 3- level $50 \mathrm{~Hz}$ AC voltage using 3 - level clamped diode inverter. The $3-$ phase voltages are filtered by using inductances to produce pure sine wave signal. It can be notice that a unity power factor between the phase voltage and line current is achieved. Minimization of the cost function in eq. (17) produced the voltage balance between the input capacitors of the 3-level diode clamped inverter in the output stage as shown in Fig. (16).

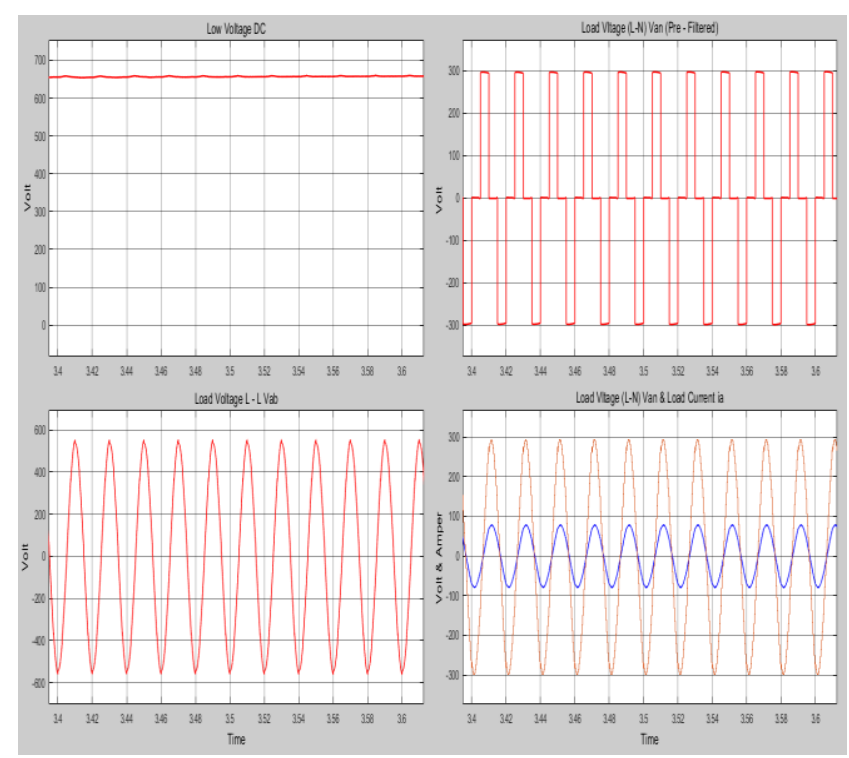

Fig. 15: Behavior of the Output Stage. 


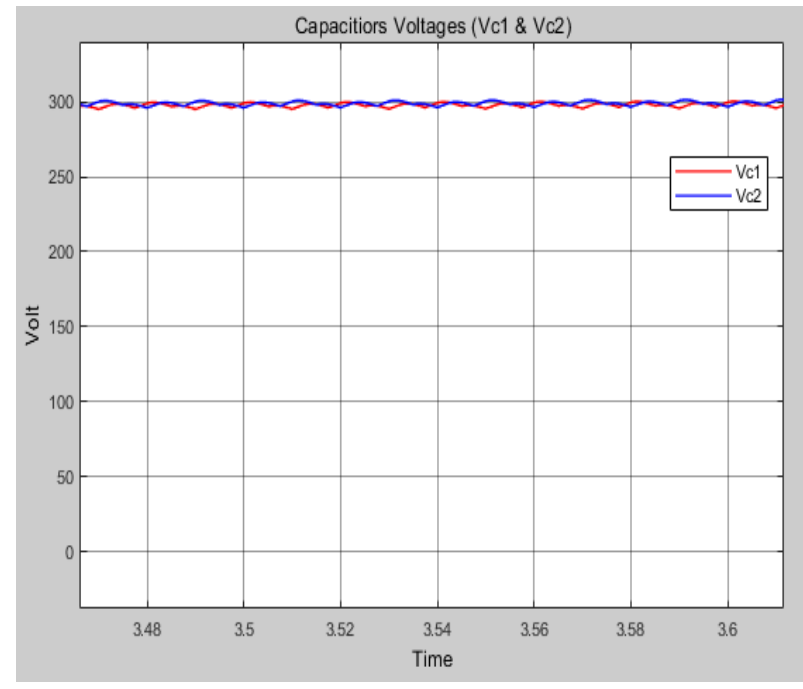

Fig. 16: Capacitors Voltages of the Output Stage.

\section{Conclusion}

In this paper, three stages solid-state transformer has been designed and implemented using Matlab/Simulink software package. Model Predictive Current Control (MPCC) is employed for driving the three stages of the SST. MPCC don't need any type of modulator and the gating signals are produced directly when the given cost function is minimized. The proposed structure of the SST performs the same task as the conventional transformer. It was shown that the designed SST provides the desired enhancement of the power factor (both in supply and load sides) and the Total Harmonic Distortion (THD). It is also providing a power of different formats with any desired frequency. Therefore, SST can be considered as a promising alternative of the conventional transformer.

\section{References}

[1] E. R. Ronan, S. D. Sudhoff, S. F. Glover and D. L. Galloway, "A Power Electronic-Based Distribution Transformer," IEEE TRANSACTIONS ON POWER DELIVERY, vol. 17, no. 2, pp. 537 - 543, 2002. https://doi.org/10.1109/61.997934.

[2] T. Zhao, L. Yang, J. Wang and A. Huang, "270 kVA Solid State Transformer Based on $10 \mathrm{kV} \mathrm{SiC} \mathrm{Power} \mathrm{Devices,"} \mathrm{in} 2007$ IEEE Electric Ship Technologies Symposium, Arlington, VA, USA, 2007. https://doi.org/10.1109/ESTS.2007.372077.

[3] M. Sabahi, S. Hosseini, M. Sharifian, A. Goharrizi and G. Gharehpetian, "Zero-voltage switching bi-directional power electronic transformer," IET Power Electronics, vol. 3, no. 5, pp. 818-828, 2008. https://doi.org/10.1049/iet-pel.2008.0070

[4] S. Xu, A. Huang and R. Burgos, "Review of Solid-State Transformer Technologies and Their Application in Power Distribution Systems," IEEE JOURNAL OF EMERGING AND SELECTED TOPICS IN POWER ELECTRONICS, vol. 1, no. 3, pp. 186-198, 2013. https://doi.org/10.1109/JESTPE.2013.2277917.

[5] X. Wang, J. Liu, S. Ouyang and F. Meng, "Research on UnbalancedLoad Correction Capability of Two Power Electronic Transformer Topologies," IEEE Transactions on Power Electronics, vol. 30, no. 6 , pp. $3044 \quad 3056, \quad 2015$. https://doi.org/10.1109/TPEL.2014.2331701.

[6] G. V. Chirkova and G. S. Zinoviev, "power quality coefficients for power electronic transformer," in 2016 17th International Conference of Young Specialists on Micro/Nanotechnologies and Electron Devices (EDM), Erlagol, Russia, 2016.

[7] M. López, F. Briz, M. Saeed, M. Arias and A. Rodríguez, "Comparative Analysis of Modular Multiport Power Electronic Transformer Topologies," in 2016 IEEE Energy Conversion Congress and Exposition (ECCE), Milwaukee, WI, USA, 2016.

[8] J. P. Contreras and J. M. Ramirez, "Multi-Fed Power Electronic Transformer for Use in Modern Distribution Systems," IEEE TRANSACTIONS ON SMART GRID, vol. 5, no. 3, pp. 1532-1541, 2014. https://doi.org/10.1109/TSG.2013.2293479.

[9] X. She, X. Yu, W. Fei and A. Huang, "Design and Demonstration of a 3.6-kV-120-V/10-kVA Solid-State Transformer for Smart Grid
Application," IEEE TRANSACTIONS ON POWER ELECTRONICS, vol. 29, no. 8, pp. 3982-3996, 2014. https://doi.org/10.1109/TPEL.2013.2293471.

[10] R. GAO, I. Husain, F. Wang and A. Q. Huang, "Solid-state transformer interfaced PMSG wind energy conversion system," in 2015 IEEE Applied Power Electronics Conference and Exposition (APEC), Charlotte, NC, USA, 2015.

[11] S. Ouyang, J. Liu, X. Wang, S. Song and X. Hou, "Comparison of four power electronic transformer topologies on unbalanced load correction capacity," in 2015 IEEE Energy Conversion Congress and Exposition (ECCE), Montreal, QC, Canada, 2015.

[12] J.-S. Lai, A. Maitra, A. Mansoor and F. Goodman, "Multilevel intelligent universal transformer for medium voltage applications," in Fourtieth IAS Annual Meeting. Conference Record of the 2005 Industry Applications Conference, Kowloon, Hong Kong, China, 2005

[13] G. Wang, S. Baek, J. Elliott and et.al, "Design and hardware implementation of Gen-1 silicon based solid state transformer," in 2011 Twenty-Sixth Annual IEEE Applied Power Electronics Conference and Exposition (APEC), Fort Worth, TX, USA, 2011. https://doi.org/10.1109/APEC.2011.5744766.

[14] L. Lin, Z. Lin and L. Haijun, "Research on the topology and control strategy of bidirectional DC-DC converter used in the power electronic transformer," in 12th IET International Conference on AC and DC Power Transmission (ACDC 2016), Beijing, China, 2016. https://doi.org/10.1049/cp.2016.0457.

[15] H. Li, Y. Wang and C. Yu, "Control of three-phase cascaded multilevel converter based power electronic transformer under unbalanced input voltages," in IECON 2016 - 42nd Annual Conference of the IEEE Industrial Electronics Society, Florence, Italy, 2016.

[16] S. Bifaretti, P. Zanchetta, A. Watson and et.al, "Advanced Power Electronic Conversion and Control System for Universal and Flexible Power Management," IEEE TRANSACTIONS ON SMART GRID, vol. 2, no. 2, pp. 231-243, 2011. https://doi.org/10.1109/TSG.2011.2115260.

[17] L. Wang, D. Zhang, Y. Wang and et.al, "Power and Voltage Balance Control of a Novel Three-phase Solid State Transformer Using Multilevel Cascaded H-Bridge Inverters for Microgrid Applications," IEEE Transactions on Power Electronics, vol. 31, no. 4, pp. 3289 3301, 2016. https://doi.org/10.1109/TPEL.2015.2450756.

[18] B. Fan, Y. Li, K. Wang and et.al, "Hierarchical System Design and Control of an MMC-Based Power-Electronic Transformer," IEEE Transactions on Industrial Informatics, vol. 13, no. 1, pp. 238 - 247, 2017. https://doi.org/10.1109/TII.2016.2522190.

[19] Y. Li, Y. Han, Y. Cao and et.al, "A modular multilevel converter type solid state transformer with internal model control method," Electrical Power and Energy Systems, vol. 85, pp. 153-163, 2017. https://doi.org/10.1016/j.ijepes.2016.09.001.

[20] D. Grider, M. Das, A. Agarwal and et.al, "10 kV/120 A SiC DMOSFET half $\mathrm{H}$-bridge power modules for 1 MVA solid state power substation," in 2011 IEEE Electric Ship Technologies Symposium, Alexandria, VA, USA, 2011 https://doi.org/10.1109/ESTS.2011.5770855.

[21] Q. Huang, Q. Zhu, L. Wang and et.al, "15 kV SiC MOSFET: An Enabling Technology for Medium Voltage Solid State Transformers," CPSS TRANSACTIONS ON POWER ELECTRONICS AND APPLICATIONS, vol. 2, no. 2, pp. 118-130, 2017. https://doi.org/10.24295/CPSSTPEA.2017.00012.

[22] P. Cortes, M. P. Kazmierkowski, R. M. Kennel and et.al, "Predictive Control in Power Electronics and Drives," IEEE TRANSACTIONS ON INDUSTRIAL ELECTRONICS, vol. 55, no. 12, pp. 4312-4324, 2008. https://doi.org/10.1109/TIE.2008.2007480.

[23] J. Rodriguez, P. Cortes, R. Kennel and et.al, "Model predictive control -- a simple and powerful method to control power converters," in 2009 IEEE 6th International Power Electronics and Motion Control Conference, Wuhan, China, 2009. https://doi.org/10.1109/IPEMC.2009.5289335.

[24] T. Geyer, "A Comparison of Control and Modulation Schemes for Medium-Voltage Drives: Emerging Predictive Control Concepts versus PWM-Based Schemes," IEEE TRANSACTIONS ON INDUSTRY APPLICATIONS, vol. 47, no. 3, pp. 1380-1389, 2011. https://doi.org/10.1109/TIA.2011.2127433.

[25] S. Vazquez, J. I. Leon, L. G. Franquelo and et.al, "Model Predictive Control: A Review of Its Applications in Power Electronics," IEEE Industrial Electronics Magazine, vol. 8, no. 1, pp. 16 - 31, 2014. https://doi.org/10.1109/MIE.2013.2290138.

[26] R. P. Aguilera and D. E. Quevedo, "Predictive Control of Power Converters: Designs with Guaranteed Performance," IEEE Transactions on Industrial Informatics, vol. 11, no. 1, pp. 53 - 63, 2015. https://doi.org/10.1109/TII.2014.2363933. 
[27] B. Stellato, T. Geyer and P. J. Goulart, "High-Speed Finite Control Set Model Predictive Control for Power Electronics," IEEE Transactions on Power Electronics, vol. 32, no. 5, pp. 4007 - 4020, 2017. https://doi.org/10.1109/TPEL.2016.2584678.

[28] S. Kouro, M. A. Perez, J. Rodriguez and et.al, "Model Predictive Control: MPC's Role in the Evolution of Power Electronics," IEEE Industrial Electronics Magazine, vol. 9, no. 4, pp. 8 - 21, 2015. https://doi.org/10.1109/MIE.2015.2478920.

[29] S. Falcones, X. Mao and R. Ayyanar, "Topology comparison for Solid State Transformer implementation," in IEEE PES Genera
Meeting,
Providence,
RI,
USA,
2010 . https://doi.org/10.1109/PES.2010.5590086.

[30] R. J. G. Montoya, A. Mallela and J. C. Balda, "An evaluation of selected solid-state transformer topologies for electric distribution systems," in 2015 IEEE Applied Power Electronics Conference and Exposition (APEC), Charlotte, NC, USA, 2015.

[31] X. She, R. Burgos, G. Wang and et.al, "Review of solid state transformer in the distribution system: From components to field application," in 2012 IEEE Energy Conversion Congress and Exposition (ECCE), Raleigh, NC, USA, 2012

[32] L. A. G. Rodriguez, V. Jones, A. R. Oliva and et.al, "A New SST Topology Comprising Boost Three-Level AC/DC Converters for Applications in Electric Power Distribution Systems," IEEE Journal of Emerging and Selected Topics in Power Electronics, vol. 5, no. 2, pp. 735 - 746, 2017. https://doi.org/10.1109/JESTPE.2017.2677523.

[33] A. R. Izadinia and H. R. Karshenas, "Optimized current control of vienna rectifier using finite control set model predictive control," in 2016 7th Power Electronics and Drive Systems Technologies Conference (PEDSTC), Tehran, Iran, 2016. 